\title{
Expected Number and Flux Distribution of Gamma\#Ray Burst Afterglows with High Redshifts
}

\section{Citation}

Ciardi, Benedetta, and Abraham Loeb. 2000. “Expected Number and Flux Distribution of Gamma\#Ray Burst Afterglows with High Redshifts." The Astrophysical Journal 540 (2): 687-96. https://doi.org/10.1086/309384.

\section{Permanent link}

http://nrs.harvard.edu/urn-3:HUL.InstRepos:41393258

\section{Terms of Use}

This article was downloaded from Harvard University's DASH repository, and is made available under the terms and conditions applicable to Other Posted Material, as set forth at http:// nrs.harvard.edu/urn-3:HUL.InstRepos:dash.current.terms-of-use\#LAA

\section{Share Your Story}

The Harvard community has made this article openly available.

Please share how this access benefits you. Submit a story.

Accessibility 
The AstrophysicAL JournaL, 540:687-696, 2000 September 10

(c) 2000. The American Astronomical Society. All rights reserved. Printed in U.S.A.

\title{
EXPECTED NUMBER AND FLUX DISTRIBUTION OF GAMMA-RAY BURST AFTERGLOWS WITH HIGH REDSHIFTS
}

\author{
Benedetta Ciardi ${ }^{1,2}$ and Abraham Loeb ${ }^{2}$ \\ Received 2000 February 22; accepted 2000 March 17
}

\begin{abstract}
If gamma-ray bursts (GRBs) occur at high redshifts, then their bright afterglow emission can be used to probe the ionization and metal enrichment histories of the intervening intergalactic medium during the epoch of reionization. In contrast to other sources, such as galaxies or quasars, which fade rapidly with increasing redshift, the observed infrared flux from a GRB afterglow at a fixed observed age is only a weak function of its redshift. This results from a combination of the spectral slope of GRB afterglows and the time stretching of their evolution in the observer's frame. Assuming that the GRB rate is proportional to the star formation rate and that the characteristic energy output of GRBs is $\sim 10^{52}$ ergs, we predict that there are always $\sim 15$ GRBs from redshifts $z \gtrsim 5$ across the sky that are brighter than $\sim 100$ nJy at an observed wavelength of $\sim 2 \mu \mathrm{m}$. The infrared spectrum of these sources could be taken with the future Next Generation Space Telescope as a follow-up to early X-ray localization with the Swift satellite.
\end{abstract}

Subject headings: galaxies: ISM — gamma rays: bursts — intergalactic medium

\section{INTRODUCTION}

The past decade has been marked by major observational breakthroughs concerning the properties of the gamma-ray burst (GRB) sources. The Burst and Transient Source Experiment (BATSE) on board the Compton Gamma Ray Observatory (Meegan et al. 1992) showed that the GRB population is distributed isotropically across the sky and that there is a deficiency of faint GRBs relative to a Euclidean distribution. These were the first observational clues indicating a cosmological distance scale for GRBs. The localization of GRBs by X-ray observations with the BeppoSAX satellite (Costa et al. 1997) allowed the detection of afterglow emission at optical (e.g., van Paradijs et al. 1997) and radio (e.g., Frail et al. 1997; Frail, Waxman, \& Kulkarni 2000) wavelengths up to more than a year following the events (Fruchter et al. 1999; Frail et al. 2000). The afterglow emission is characterized by a broken power-law spectrum with a peak frequency that declines with time. The radiation can be well modeled as synchrotron emission from a decelerating blast wave, created by the GRB explosion in an ambient medium, plausibly the interstellar medium of galaxies (Waxman 1997; Wijers \& Galama 1999; Mészáros 1999; but see also Chevalier \& Li 2000). The detection of spectral features, such as metal absorption lines (Metzger et al. 1997), in some optical afterglows allowed an unambiguous identification of the cosmological distance scale. By now, the redshift of almost a dozen GRBs has been identified through the detection either of absorption features in the afterglow spectra or of emission lines from host galaxies (see Fig. 3 in Kulkarni et al. 2000).

The central engine of GRBs is still unknown. Since the inferred energy release is comparable to or higher than that in supernovae, most popular models relate GRBs to stellar remnants, such as neutron stars or black holes (see, e.g., Eichler et al. 1989; Paczyński 1991, 1998; Usov 1992; Mochkovitch et al. 1993; MacFadyen \& Woosley 1999).

\footnotetext{
${ }^{1}$ Università degli Studi di Firenze, Dipartimento di Astronomia, Largo E. Fermi 5, Firenze, Italy.

${ }^{2}$ Harvard-Smithsonian Center for Astrophysics, 60 Garden Street, Cambridge, MA 02138.
}

Recently it has been claimed that the late evolution of some rapidly declining optical afterglows shows a component that is possibly associated with supernova emission (e.g., Bloom et al. 1999; Reichart 1999). If the supernova association will be confirmed by detailed spectra of future afterglows, the GRB phenomenon will be linked to the terminal evolution of massive stars.

Any association of GRBs with the formation of single compact stars implies that the GRB rate should trace the star formation history of the universe (Totani 1997; Sahu et al. 1997; Wijers et al. 1998; but see Krumholz, Thorsett, \& Harrison 1998). Owing to their high brightness, GRB afterglows might be detected out to exceedingly high redshifts. Similarly to quasars, the broadband emission of GRB afterglows can be used to probe the absorption properties of the intergalactic medium (IGM) out to the epoch when it was reionized at a redshift $z \sim 10$ (Miralda-Escudé \& Rees 1998; Loeb 1999). Lamb \& Reichart (2000) have extrapolated the observed gamma-ray and afterglow spectra of known GRBs to high redshifts and emphasized the important role that their detection might play in probing the IGM. Simple scaling of the long-wavelength spectra and temporal evolution of afterglows with redshift implies that at a fixed time lag after the GRB in the observer's frame, there is only mild change in the observed flux at infrared or radio wavelengths with increasing redshift. This results in part from the fact that afterglows are brighter at earlier times and that a given observed time refers to an earlier intrinsic time in the source frame as the source redshift increases. The mild dependence of the long-wavelength flux on redshift is in contrast with other high-redshift sources such as galaxies or quasars, which fade rapidly with increasing redshift (Haiman \& Loeb 1998, 1999). The "apparent brightening" of GRB afterglows with redshift could be further enhanced by the expected increase in the mean density of the interstellar medium of galaxies at increasing redshifts (Wood \& Loeb 1999).

It therefore appears natural to use GRBs as an important tool in probing the high-redshift universe and its star formation history (Blain \& Natarajan 2000). Since GRBs are rare, all-sky searches for their early gamma-ray emission are 
needed before follow-up observations at much longer wavelengths are conducted.

In this paper we model the emission from GRB afterglows and follow their number counts at different wavelengths as a function of redshift. For simplicity, we assume that the ambient gas surrounding GRB sources is the interstellar medium of their host galaxies. In $\S 2$ we review our model for both the relativistic and nonrelativistic stages of the afterglow emission, including a simple prescription for the redshift evolution of the interstellar medium of galaxies (see the Appendix for details). The numerical results and their dependence on model assumptions are discussed in $\S 3$. Finally, $\S 4$ summarizes our main conclusions and describes the implications of our results.

\section{AFTERGLOW EMISSION}

\subsection{Relativistic Regime}

GRB afterglows can be modeled as synchrotron emission from a decelerating relativistic blast wave, created by the GRB explosion in an external medium. For a point explosion in a uniform medium, the shock structure in the highly relativistic regime is described by the Blandford \& McKee (1976) self-similar solution. The synchrotron emission originates from a power-law population of shock-accelerated electrons in a strong magnetic field (Waxman 1997; Mészáros, Rees, \& Wijers 1998; Sari, Piran, \& Narayan 2000), with both the electrons and the magnetic field having close to equipartition energy densities (see Medvedev \& Loeb 1999 for the possible origin of the magnetic field). For simplicity, we focus our discussion on a spherically symmetric explosion in a uniform ambient medium, assumed to be the interstellar medium of the host galaxy, although at least some GRB blast waves might be expanding into the stellar wind of the progenitor star (Dai \& $\mathrm{Lu} 1998$; Mészáros et al. 1998; Chevalier \& Li 2000). The dependence of our results on the ambient medium density will be discussed in $\S 3.3$. We assume that the shock-accelerated electrons have a power-law distribution of Lorentz factor $\gamma_{e}$ with a minimum Lorentz factor $\gamma_{m}$ (see, e.g., Sari et al. 1998). We also define $\gamma_{c}$ as the threshold Lorentz factor below which electrons do not lose a significant fraction of their energy to radiation. In the fast-cooling regime, when $\gamma_{m}>$ $\gamma_{c}$, all of the electrons cool rapidly down to a Lorentz factor $\sim \gamma_{c}$ and the flux observed at the frequency $v$ is given by

$$
F_{v}=F_{v_{m}} \begin{cases}\left(v / v_{c}\right)^{1 / 3} & v_{c}>v, \\ \left(v / v_{c}\right)^{-1 / 2} & v_{m}>v \geq v_{c}, \\ \left(v_{m} / v_{c}\right)^{-1 / 2}\left(v / v_{m}\right)^{-p / 2} & v \geq v_{m},\end{cases}
$$

where $F_{v_{m}}$ is the observed peak flux; $v_{c}=v\left(\gamma_{c}\right)$ and $v_{m}=$ $v\left(\gamma_{m}\right)$ are the characteristic synchrotron frequency calculated at $\gamma_{c}$ and $\gamma_{m}$, respectively; and $p$ is the power-law index of the electron energy distribution. A typical value of $p \approx 2.5$ often fits both the GRB and the afterglow observations (Sari et al. 1998; Kumar \& Piran 2000).

When $\gamma_{c}>\gamma_{m}$, only electrons with $\gamma_{e}>\gamma_{c}$ cool efficiently. In this slow-cooling regime, the observed flux is

$$
F_{v}=F_{v_{m}} \begin{cases}\left(v / v_{m}\right)^{1 / 3} & v_{m}>v, \\ \left(v / v_{m}\right)^{-(p-1) / 2} & v_{c}>v \geq v_{m}, \\ \left(v_{c} / v_{m}\right)^{-(p-1) / 2}\left(v / v_{c}\right)^{-p / 2} & v \geq v_{c} .\end{cases}
$$

Typically, synchrotron self-absorption results in an additional break, at a frequency $\lesssim 5 \mathrm{GHz}$. We will not consider the low-frequency regime below this break in our discussion. Assuming a fully adiabatic shock (Sari et al. 1998),

$$
\begin{gathered}
v_{c}=2.7 \times 10^{12} \epsilon_{B}^{-3 / 2} E_{52}^{-1 / 2} n_{1}^{-1} t_{d}^{-1 / 2} \times(1+z)^{-1 / 2} \mathrm{~Hz}, \\
v_{m}=5.7 \times 10^{14} \epsilon_{B}^{1 / 2} \epsilon_{e}^{2} E_{52}^{1 / 2} t_{d}^{-3 / 2}(1+z)^{1 / 2} \mathrm{~Hz}, \\
F_{v_{m}}=1.1 \times 10^{5} \epsilon_{B}^{1 / 2} E_{52} n_{1}^{1 / 2} d_{28}^{-2}(1+z) \mu \mathrm{Jy} .
\end{gathered}
$$

The redshift dependence results from the fact that the radiation emitted by a source at a redshift $z$ at the frequency $v_{s}$ over a time $\Delta t_{s}$, will be observed at $z=0$ at a frequency $v_{0}=v_{s} /(1+z)$ over a time $\Delta t_{0}=(1+z) \Delta t_{s}$. Here $\epsilon_{B}$ and $\epsilon_{e}$ are the fraction of the shock energy that is converted to magnetic fields and accelerated electrons, respectively, for which we adopt the values $\epsilon_{B}=0.1$ and $\epsilon_{e}=0.2$ (see, e.g., Waxman 1997); $E=E_{52} 10^{52}$ ergs is the energy of the spherical shock; $n=n_{1} \times 1 \mathrm{~cm}^{-3}$ is the mean number density of the ambient gas; $t=t_{d} \times 1$ day is the time lag since the GRB trigger, as measured in the observer frame, and $d_{L}=d_{28} 10^{28} \mathrm{~cm}$ is the cosmology-dependent luminosity distance. For a flat universe $\left(\Omega_{0}=\Omega_{m}+\Omega_{\Lambda}=1\right)$, the luminosity distance can be written as

$$
d_{L}=(1+z) \int_{0}^{z}\left(1+z^{\prime}\right)\left|\frac{d t}{d z^{\prime}}\right| d z^{\prime},
$$

where

$$
\left(\frac{d t}{d z}\right)^{-1}=-H_{0}(1+z) \sqrt{\left(1+\Omega_{m} z\right)(1+z)^{2}-\Omega_{\Lambda}\left(2 z+z^{2}\right)} .
$$

The current Hubble constant is $H_{0}=100 h \mathrm{~km} \mathrm{~s}^{-1} \mathrm{Mpc}^{-1}$. Throughout the paper we adopt a flat cosmology with $h=0.65$, density parameters $\Omega_{m}=0.35$ and $\Omega_{\Lambda}=0.65$, and a cold dark matter power spectrum of density fluctuations with power-law index $n=0.96$, an amplitude $\sigma_{8}=0.87$, and baryon density parameter $\Omega_{b}=0.04$ (Bahcall et al. 1999). The explosion energy, $E$, is highly uncertain. Based on X-ray afterglow data, Freedman \& Waxman (1999) inferred explosion energies in the range $\left(10^{51.5}-10^{53.5} \mathrm{ergs}\right)(\Delta \Omega / 4 \pi)$, where $\Delta \Omega$ is the solid angle into which the energy is channeled. If one assumes that GRB explosions are isotropic, then in some events the radiated energy is estimated to be in excess of $10^{53}$ ergs, reaching a value of $3.4 \times 10^{54} \mathrm{ergs}$ for GRB 990123 (see, e.g., Kulkarni et al. 1999). It has been suggested that these values are reduced by orders of magnitude due to beaming (Kulkarni et al. 1999; Mészáros \& Rees 1999), although the relatively low efficiency for converting internal shock energy into radiation might imply an energy budget as high as $10^{54}$ ergs even after the beaming correction (Kumar 1999). Unless otherwise noted, we adopt the value $E=10^{52} \mathrm{ergs}$, which is intermediate for the range inferred by Freedman \& Waxman (1999) (see also Böttcher $\&$ Dermer 2000, and references therein, for a discussion on the possible broad distribution of explosion energies and the corresponding increase in the event rate). In $\S 3.3$ we will discuss the effect of beaming and different choices of the explosion energy on our results.

\subsection{Nonrelativistic Regime}

As the blast wave decelerates, the fireball eventually makes a transition from relativistic to subrelativistic (sr) 
expansion (Huang, Dai, \& Lu 1998; Woods \& Loeb 1999; Frail et al. 2000; Wei \& Lu 1999). In this regime, the evolution of the GRB remnants is well described by the SedovTaylor solution (Taylor 1950; Sedov 1959). The time when this transition takes place in the observer frame, $t_{\mathrm{sr}}$, can be approximately derived by setting the shock velocity in the Sedov-Taylor solution to be equal to the speed of light, $c$. This gives

$$
\begin{aligned}
t_{\mathrm{sr}} & =(1+z)\left[\frac{E}{m_{p} n}\left(\frac{0.47}{c}\right)^{5}\right]^{1 / 3} \\
& =1.8 \times 10^{2}(1+z) E_{52}^{1 / 3} n_{1}^{-1 / 3} \text { days } .
\end{aligned}
$$

In the subrelativistic regime, the shock radius and velocity evolve as

$$
\begin{gathered}
r(t)=r\left(t_{\mathrm{sr}}\right)\left(t / t_{\mathrm{sr}}\right)^{2 / 5}, \\
v(t)=c\left(t / t_{\mathrm{sr}}\right)^{-3 / 5},
\end{gathered}
$$

where all times are in the observer frame and $r\left(t_{\mathrm{sr}}\right)$ is the shock radius at time $t_{\mathrm{sr}}$. For a strong nonrelativistic shock, the postshock particle density, energy density, and magnetic field are

$$
\begin{gathered}
n^{\prime}=4 n, \\
u^{\prime}=\frac{9}{8} n m_{p} v^{2}, \\
B^{\prime}=\left(8 \pi \epsilon_{B} u^{\prime}\right)^{1 / 2} .
\end{gathered}
$$

The observed flux requires a substitution of the values of $v_{c}$, $v_{m}$, and $F_{v_{m}}$ in the subrelativistic regime into equations (1) and (2). The synchrotron formulae can be used since the emitting electrons are still ultrarelativistic for the relevant remnant ages in this regime. The characteristic synchrotron frequency is given by

$$
v(\gamma)=\gamma^{2} \frac{e B^{\prime}}{2 \pi m_{e} c} .
$$

Recalling that $\gamma_{m} \propto B^{\prime 2} \propto u^{\prime} / n^{\prime}$, we find $\gamma_{m} \propto v^{2} \propto t^{-6 / 5}$, and $\gamma_{c} \propto\left(B^{\prime 2} t\right)^{-1} \propto t^{1 / 5}$. Equation (14) then provides an expression for $v_{c}$ and $v_{m}$ in the subrelativistic regime. In particular, $v_{c} \propto t^{-1 / 5}$ and $v_{m} \propto t^{-3}$. During the relativistic expansion, the observed peak flux is constant in time; however, in the subrelativistic regime it varies as $F_{v_{m}} \propto r^{3} B^{\prime} \propto t^{3 / 5}$.

In the UV regime one needs to take account of absorption by the IGM. At redshifts greater than the reionization redshift, $z_{\text {reion }}$, the neutral IGM is optically thick to photon energies above the Ly $\alpha$ resonance. Recent models for the reionization of the IGM predict $z_{\text {reion }}$ in the range of 7-12 (Haiman \& Loeb 1997; Miralda-Escudé, Haehnelt, \& Rees 2000; Valageas \& Silk 1999; Chiu \& Ostriker 2000; Gnedin 2000; Ciardi et al. 2000). Here we adopt $z_{\text {reion }}=8$, the precise choice of which has only a minor effect on our results since the Ly $\alpha$ forest yields a high opacity at the redshifts of interest even if most of the IGM is ionized. For $z \lesssim 5$ we use the updated Ly $\alpha$ forest opacity derived by Haardt \& Madau (1996) and Madau, Haardt, \& Rees (1999), which is based on the observed absorber distribution in the spectra of high-redshift quasars. The continuum depression blueward of the Ly $\alpha$ resonance is already close to unity at $z \sim 5$ (see Fig. 13 in Stern \& Spinrad 1999), and so the treatment of the Ly $\alpha$ absorption at $5 \lesssim z \lesssim 8$ has little effect on our results.
An expression for the ambient gas density as a function of redshift is derived in the Appendix. For simplicity, we assume that the ambient gas has the mean density of the interstellar medium of the host galaxy, as inferred for GRB 970508 and GRB 971214 (Waxman 1997; Wijers \& Galama 1999).

\section{RESULTS}

\subsection{Flux Evolution}

Figure 1 shows the observed flux for a GRB hosted by the average halo mass as a function of redshift. This average is calculated based on the Press \& Schechter (1974) formalism, which provides the mass function of dark matter halos as a function of redshift. The two sets of curves correspond to two observed wavelengths: in Figure $1 a$ the thick lines correspond to $\lambda_{\text {obs }}=2 \mu \mathrm{m}$ and the thin lines to $5000 \AA$, while in Figure $1 b$ the thick lines correspond to $\lambda_{\text {obs }}=10 \mathrm{~cm}$ and the thin lines to $1 \mathrm{~mm}$. Within each set, the lines correspond to an observed time of $1 \mathrm{hr}$ (solid lines), 1 day (dotted lines), and 10 days (dashed lines). While the optical and infrared emission peak at observed times shorter than an hour, the radio emission has not reached its maximum even after 50 days.

At observed frequencies below the Ly $\alpha$ resonance frequency, $v_{\alpha}(z)=2.47 \times 10^{15} /(1+z) \mathrm{Hz}$, the emitted flux is not absorbed by the intervening intergalactic medium at a redshift $z$. Figure 1 shows that at these frequencies the afterglow flux is only weakly dependent on redshift. This can be crudely understood from the scaling laws implied by equations (1) and (2) by considering the simple Einstein-de Sitter cosmology $\left(\Omega_{m}=1\right)$, for which $d_{L} \propto(1+z)[1-$ $\left.(1+z)^{-1 / 2}\right]$. Recalling that $n \propto(1+z)^{4}$ for a fixed host galaxy mass (see eqs. [A4]-[A7]) and substituting these scaling laws into equations (1) and (2), we find for the case of fast cooling

$$
F_{v} \propto \begin{cases}(1+z)^{5 / 2}\left[1-(1+z)^{-1 / 2}\right]^{-2} & v_{c}>v \\ (1+z)^{-5 / 4}\left[1-(1+z)^{-1 / 2}\right]^{-2} & v_{m}>v \geq v_{c} \\ (1+z)^{(p-6) / 4}\left[1-(1+z)^{-1 / 2}\right]^{-2} & v \geq v_{m}\end{cases}
$$

while for slow cooling

$$
F_{v} \propto \begin{cases}(1+z)^{5 / 6}\left[1-(1+z)^{-1 / 2}\right]^{-2} & v_{m}>v \\ (1+z)^{(p-3) / 4}\left[1-(1+z)^{-1 / 2}\right]^{-2} & v_{c}>v \geq v_{m} \\ (1+z)^{(p-6) / 4}\left[1-(1+z)^{-1 / 2}\right]^{-2} & v \geq v_{c}\end{cases}
$$

Although these scaling laws ignore the evolution of the characteristic galaxy mass with redshift, they demonstrate that the observed flux does not decline rapidly with increasing redshift but rather might increase with redshift at low frequencies (e.g., in the millimeter or radio regimes). This behavior results from three causes: (1) for a fixed observed time lag after the GRB, the cosmic time dilation implies that the higher the source redshift is the earlier the emission time is and the brighter the intrinsic afterglow luminosity is; (2) the spectral slope of GRB afterglows results in a favorable $K$-correction for the redshifts and wavelengths of interest; and (3) the gas density in galaxies increases with redshift (due to the corresponding increase in the mean density of the universe).

\subsection{Number Count}

In a snapshot mode of observations, the total number of GRBs with observed flux greater than $F$ at an observed 

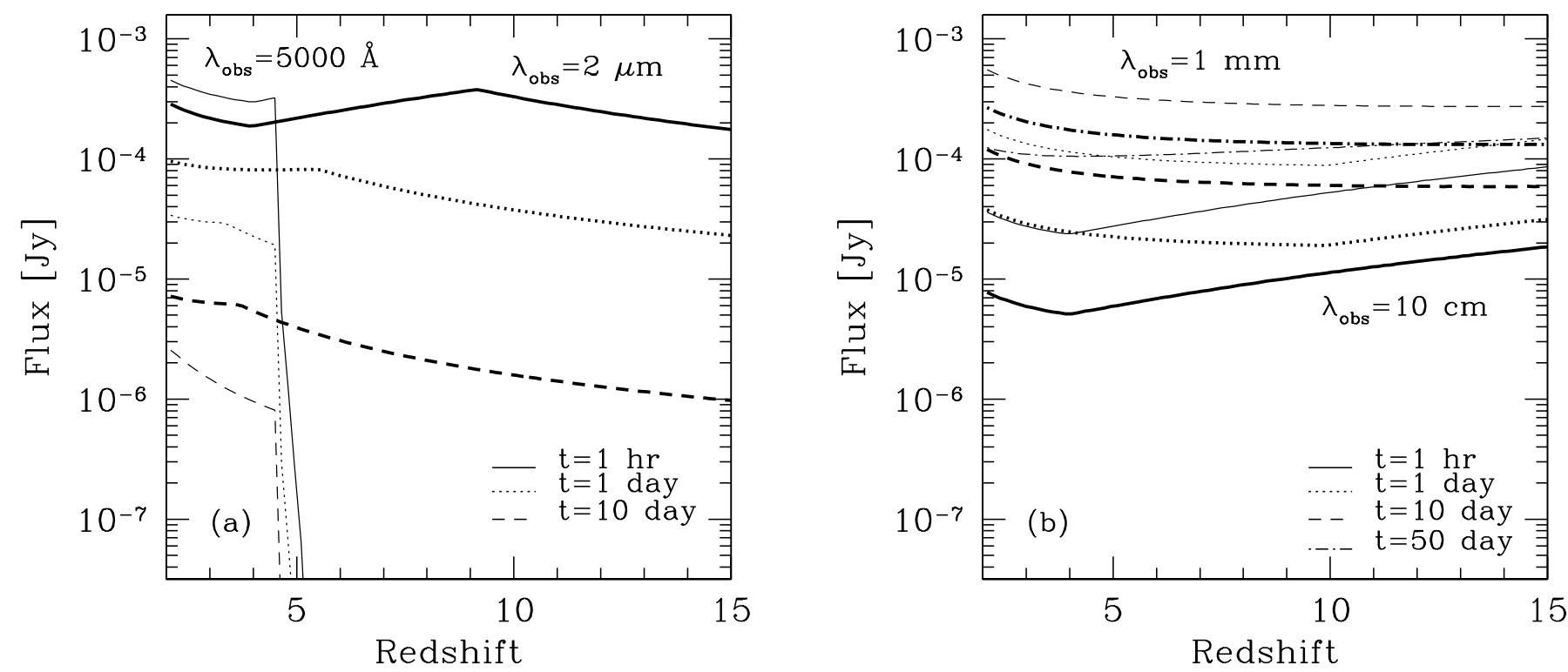

FIG. 1. - (a) Observed flux for a GRB hosted by a "typical" halo (having the mean mass) as a function of redshift (see text for details). The curves are for a frequency $v=6 \times 10^{14} \mathrm{~Hz}\left(\lambda_{\text {obs }}=5000 \AA\right.$, thin lines $)$ and $v=1.5 \times 10^{14} \mathrm{~Hz}\left(\lambda_{\text {obs }}=2 \mu \mathrm{m}\right.$, thick lines $)$. From the top to the bottom, different observed times after the GRB are shown: $1 \mathrm{hr}$ (solid line), 1 day (dotted line), and 10 days (dashed line). (b) As in (a) but for $v=3 \times 10^{11} \mathrm{~Hz}\left(\lambda_{\text {obs }}=1 \mathrm{~mm}\right.$, thin lines) and $v=3 \times 10^{9} \mathrm{~Hz}\left(\lambda_{\mathrm{obs}}=10 \mathrm{~cm}\right.$, thick lines $)$. The dot-dashed line corresponds to an observed time of 50 days.

frequency $v$ is

$$
\begin{aligned}
N(>F, v)= & \int_{0}^{\infty} \int_{M_{\min }}^{\infty} r_{\mathrm{GRB}}(z, M) \frac{t_{F}(z, M, v)}{(1+z)} \\
& \times \frac{d n}{d M}(z) \frac{d V}{d z} d M d z,
\end{aligned}
$$

where $r_{\mathrm{GRB}}$ denotes the GRB rate in a galaxy halo of mass $M$ at a redshift $z, t_{F}$ is the observed time over which a GRB event from this halo yields a flux brighter than $F$ at a frequency $v, d n / d M$ is the comoving number density of halos with masses between $M$ and $M+d M$ (based on the PressSchechter formalism), and $M_{\min }$ is the minimum galaxy mass in which stars form at a redshift $z$. The minimum halo mass inside which star formation occurs is related to a minimum virial temperature of $\sim 10^{4} \mathrm{~K}$ below which atomic cooling of the gas is suppressed and fragmentation into stars is inhibited (Haiman, Rees, \& Loeb 1997; Ciardi et al. 2000). This minimum virial temperature implies $M_{\text {min }}=4.4 \times 10^{9} M_{\odot}(1+z)^{-1.5} h^{-1}$ (Padmanabhan 1993) and is also consistent with our choice for the sound speed of the disk (see the Appendix). The comoving volume element per unit redshift, $d V / d z$, is given by

$$
\frac{d V}{d z}=\frac{4 \pi c d_{L}^{2}}{(1+z)}\left|\frac{d t}{d z}\right|
$$

where $d_{L}$ and $d t / d z$ are given in equations (6) and (7), respectively. Next we consider the value of the GRB rate, $r_{\mathrm{GRB}}$.

If GRBs are related to the final stages in the evolution of massive stars, then one may assume that the GRB rate, $r_{\mathrm{GRB}}$, is proportional to the star formation rate (SFR). This assumption is justified as long as the time delay between the formation of a massive star and a GRB event is short compared to the Hubble time at the redshift of interest. We adopt this as our working hypothesis. Wijers et al. (1998) have estimated the constant of proportionality between $r_{\mathrm{GRB}}$ and the cosmic SFR for an Einstein-de Sitter cosmol- ogy, but their results do not change significantly for other values of the cosmological parameters (J. Bagla 1999, private communication). Using the observed redshift history of the SFR per comoving volume in the universe (Lilly et al. 1996; Madau et al. 1996) and the observed distribution of gamma-ray flux of GRBs, Wijers et al. (1998) have calibrated the GRB rate per comoving volume. Assuming that GRBs are standard candles, they have found a GRB rate at $z=0$ of $R_{\mathrm{GRB}}(0)=0.14 \pm 0.02 \mathrm{Gpc}^{-3} \mathrm{yr}^{-1}$. This estimate ignores recent corrections to the cosmic SFR. The same calculation, performed with an SFR per comoving volume that flattens at high redshift, as suggested by more recent corrections for dust extinction, still leads to similar results (J. Bagla 1999, private communication). Assuming that the proportionality constant does not vary with redshift, we write

$$
r_{\mathrm{GRB}}(z, M)=\frac{R_{\mathrm{GRB}}(0)}{\dot{\rho}_{*}(0)} \dot{M}_{*}(z, M) .
$$

Here $\dot{\rho}_{*}(0)$ is the star formation rates per comoving volume in units of $M_{\odot} \mathrm{yr}^{-1} \mathrm{Mpc}^{-3}$, and $\dot{M}_{*}(z, M)=\alpha(z) \Omega_{b} M$ is the SFR within a particular halo in units of $M_{\odot} \mathrm{yr}^{-1}$. We calibrate the star formation efficiency as a function of redshift, $\alpha(z)$, so as to match the observed cosmic SFR, $\dot{\rho}_{*}$, at $z \lesssim 4$,

$$
\dot{\rho}_{*}(z)=\int_{M_{\min }}^{\infty} \dot{M}_{*}(z) \frac{d n}{d M}(z) d M .
$$

An analytical fit for the observed $\dot{\rho}_{*}(z)$ (assuming a Salpeter initial mass function and an extinction correction of $A_{1500}=1.2 \mathrm{mag}$ ) was derived by Madau \& Pozzetti (2000):

$$
\dot{\rho}_{*}(z)=\frac{0.23 e^{3.4 z}}{e^{3.8 z}+44.7} M_{\odot} \mathrm{yr}^{-1} \mathrm{Mpc}^{-3} .
$$

At $z>4$ the SFR within a particular halo is calculated using the common prescription $\alpha(z) \propto t_{\mathrm{dyn}}^{-1}(z)$, where $t_{\mathrm{dyn}}(z)$ is the mean dynamical time of the halo. The constant of pro- 
portionality is chosen so as to match the observed cosmic SFR at $z=4$ (see Ciardi et al. 2000 for more details).

Figure 2 presents the total number counts based on equation (17). The set of curves corresponds to different observed wavelengths. From the right to the left, $\lambda_{\text {obs }}$ is equal to 10 $\mathrm{cm}, 1 \mathrm{~mm}, 2 \mu \mathrm{m}$, and $5000 \AA$. At low fluxes the number counts approach an asymptotic value. Out of the entire population of faint GRB afterglows, we predict that there are $\sim 15$ GRBs from redshifts $z \gtrsim 5$ across the sky that are brighter than $\sim 100 \mathrm{nJy}$ at an observed wavelength of $\sim 2$ $\mu \mathrm{m}$. Figure 3 illustrates the contribution to the total number counts from different redshift bins of width 0.1 , centered at $z=2$ (Fig. $3 a$ ), 6 (Fig. $3 b$ ), and 10 (Fig. 3c). Infrared or radio afterglows are detectable out to redshifts as high as 10 , while optical afterglows are strongly absorbed by the intervening IGM at $z \gtrsim 5$. Figure 4 shows the differential number count distribution per logarithmic flux interval and redshift interval of all sources at a given redshift. At high frequencies, for which the observed flux decreases with increasing redshift, low-redshift events dominate the counts at most fluxes. However, at low frequencies (i.e., millimetric wavelength) the high-redshift events dominate the counts at sufficiently low fluxes.

\subsection{Dependence of Results on Model Assumptions}

Next we consider the dependence of our results on various model parameters. Figure 5 shows the observed flux at $\lambda_{\text {obs }}=2 \mu \mathrm{m}$ and $t=1$ day for different choices of the mean ambient density. The standard case with $P_{\text {GRB }} \propto n^{2}$ (solid line) is compared to two other cases, one where $P_{\mathrm{GRB}} \propto n$ (dot-dashed line) and the other where the ambient density is kept constant and equal to $1 \mathrm{~cm}^{-3}$ (dotted line). The figure indicates that for observations in the infrared all three cases provide identical results at $z \gtrsim 5$ when one enters a regime where the observed flux becomes independent of the ambient density (either the fast-cooling regime with $v>v_{m}$ or the slow-cooling regime with $v>v_{c}$, given by eqs. [1] and [2]). Outside this regime the observed flux is

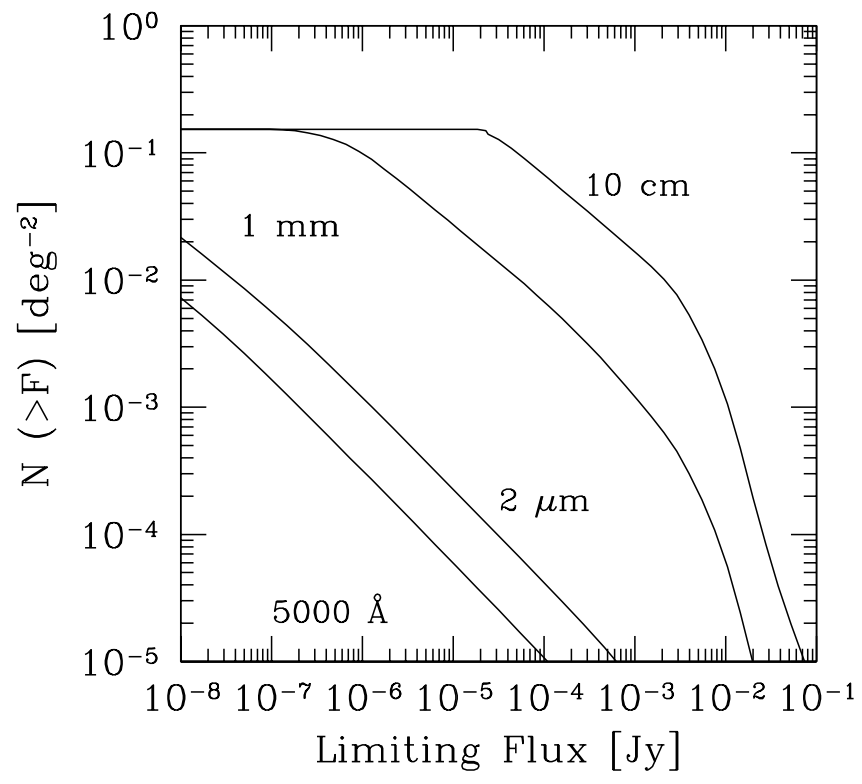

FIG. 2.-Total number of GRBs with observed flux greater than a limiting flux $F$ at different observed wavelength $\lambda_{\mathrm{ogs}}$. From the right to the left, $\lambda_{\text {obs }}$ is equal to $10 \mathrm{~cm}, 1 \mathrm{~mm}, 2 \mu \mathrm{m}$, and $5000 \mathrm{~A}$. roughly proportional to $n^{5 / 6}$ and $\sqrt{n}$ in the fast- and slowcooling cases, respectively. In the slow-cooling regime, for example, the condition $v \geq v_{c}$ leads to a minimum redshift above which the flux is independent of ambient density $(1+z) \geq 0.32 E_{52}^{-1} n_{1}^{-2} t_{d}^{-1}\left(\lambda_{\text {obs }} / 2 \mu \mathrm{m}\right)^{2}$. Hence, at long wavelengths or very low densities, the density-independent regime is not reached. Figure $5 a$ shows that the difference between the $P_{\mathrm{GRB}} \propto n^{2}$ and $P_{\mathrm{GRB}} \propto n$ cases is small since they follow a similar evolution of the gas density with redshift. Figure $5 b$ shows the number count of GRBs per logarithmic redshift interval at $z=6$, for both the standard case with $P_{\mathrm{GRB}} \propto n^{2}$ (solid line) and the case of a constant ambient density equal to $1 \mathrm{~cm}^{-3}$ (dotted line). The difference between these cases is small.

Figure 6 shows the dependence on the energy output of the afterglow number count from a redshift bin of width 0.1 centered at $z=6$ for an observed wavelength of $2 \mu \mathrm{m}$. We consider two cases that bracket the standard case $\left(E=10^{52}\right.$ ergs), namely, $E=10^{53}$ ergs (dotted line) and $E=5 \times 10^{50}$ ergs (dashed line). The increase in the observed flux as the energy output increases (see eqs. [1]-[5]) is reflected in the afterglow number count.

So far we have assumed that the GRB explosions are spherically symmetric. If, however, the energy release is beamed, then the afterglow light curve is expected to evolve differently than we assumed. At early times, as long as the expansion Lorentz factor $\gamma$ is still larger than the inverse of the jet opening angle $\theta^{-1}$, the expansion behaves as if it were spherically symmetric with the same energy output per solid angle. Once the jet decelerates to a Lorentz factor $\gamma \lesssim \theta^{-1}$, the light curve declines more rapidly since the jet starts to expand sideways and to reduce the mean energy output per solid angle (Rhoads 1999a, 1999b; Panaitescu \& Mészáros 1999). Finally, the isotropization of the energy ends when the expansion becomes subrelativistic, at which time the light curve recovers the spherically symmetric Taylor-Sedov evolution for the actual total energy output. (Synchrotron radiation losses are usually negligible in the late afterglow phase.) For an observing point that is aligned with the GRB jet axis, the afterglow light curve may start with an isotropic-equivalent energy of $E$ and end in the nonrelativistic regime with the actual energy output of $E_{\text {sr }}=\eta E$, where $\eta=(\Delta \Omega / 4 \pi)=2\left(\pi \theta^{2} / 4 \pi\right) \leq 1$ is the fraction of sky around the GRB source that is illuminated by two opposing jets of angular radius $\theta$. Hence, the afterglow makes a transition between the light curve corresponding to a highly relativistic explosion with a total energy $E$ to the light curve of a nonrelativistic explosion with an energy $\eta E$. To examine the effect of beaming on our results, we model this transition using a linear interpolation between the two light curves. We start with an isotropic equivalent of $E=10^{53}$ ergs and adopt $\theta=0.1$ so that the actual energy output is $E_{\mathrm{sr}}=5 \times 10^{50}$ ergs (since $\gamma \gtrsim 10^{2} \gg \theta^{-1}$, initially). As expected, the afterglow fluxes and source number counts make a transition between their values for the two boundary energies. Figures $6 a-6 c$ show the transition between the two regimes with a dot-dashed line. We find that, for $\lambda_{\text {obs }}=2 \mu \mathrm{m}$, the number counts are dominated by sources in the relativistic phase. However, at longer wavelengths, $\lambda_{\text {obs }}=1 \mathrm{~mm}$ or $10 \mathrm{~cm}$ (Fig. $6 b$ or Fig. $6 c$, respectively), the bulk of the emission takes place at late times when the expansion is in its mildly or subrelativistic phase. Obviously, the effect of beaming can be recovered from the shape of the afterglow light curve in individual 

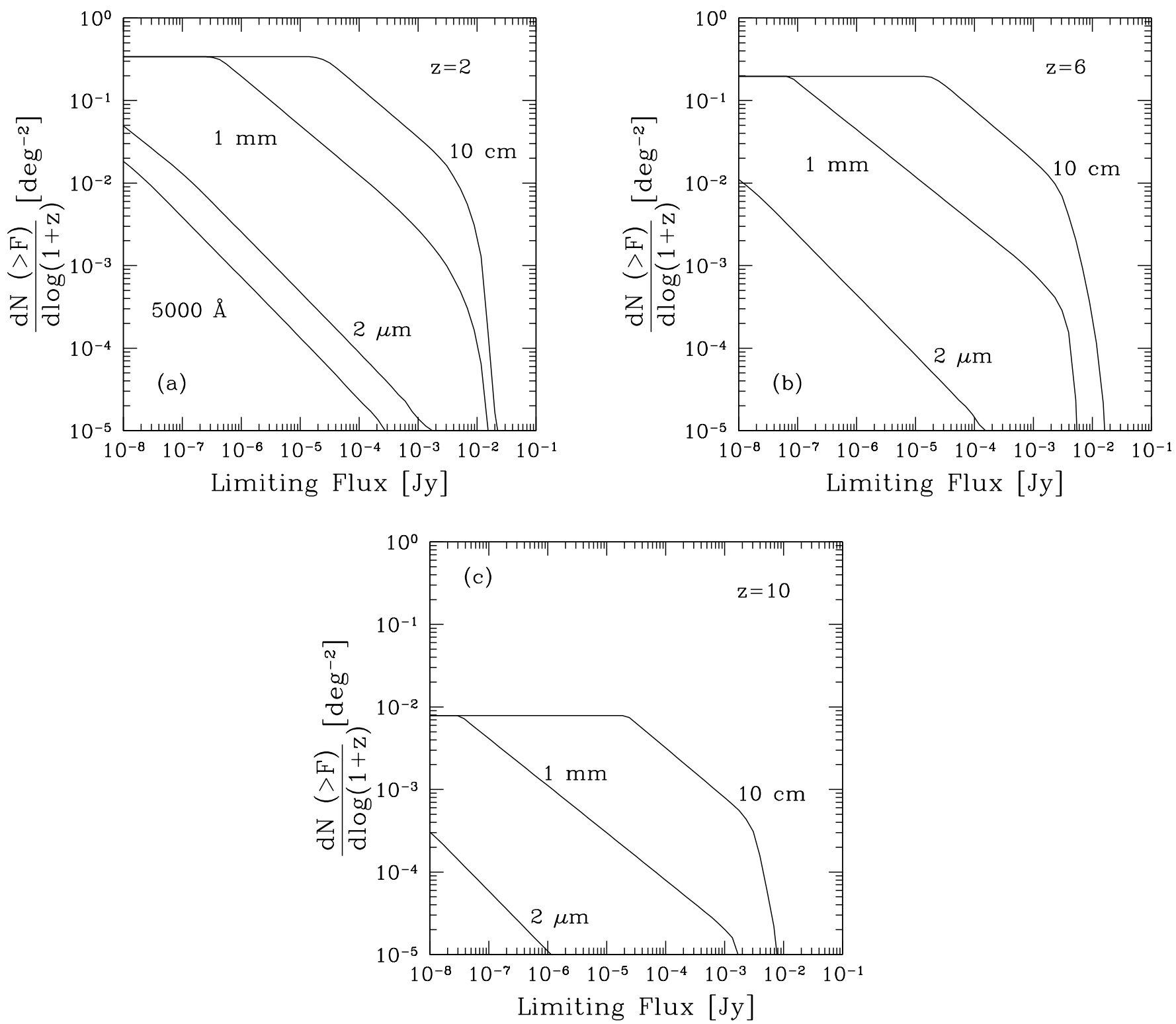

FIG. 3.-(a) Contribution to the total number count (Fig. 2) of GRBs from a redshift bin of width 0.1 centered at $z=2$. The observed wavelengths are, from the right to the left, $\lambda_{\text {obs }}=10 \mathrm{~cm}, 1 \mathrm{~mm}, 2 \mu \mathrm{m}$, and $5000 \AA$. (b) As in $(a)$ with a redshift bin of width 0.1 centered at $z=6$. (c) As in $(a)$ with a redshift bin of width 0.1 centered at $z=10$.

GRB events. Figure 6 shows that the existence of beaming might also be inferred from the number count statistics.

\section{DISCUSSION}

We have calculated the expected fluxes and number counts of high-redshift GRB afterglows, assuming that their rate of occurrence is proportional to the star formation rate. We have computed the observed properties of GRB light curves at different wavelengths ranging from the optical to the radio, treating both the relativistic and subrelativistic phases of the expansion of their remnants.

Our main result is that at a fixed observed time after the GRB event, the characteristic afterglow flux is not decreasing rapidly with increasing redshift (Figs. $1 a$ and $1 b$ ), in contrast with other high-redshift sources, such as galaxies or quasars. Hence, the broadband spectrum of GRB afterglows is ideally suited for probing the ionization state and metal content of the intergalactic medium at high redshifts, particularly during the epoch of reionization (Loeb 1999; Lamb \& Reichart 2000).

The only difficulty in using GRBs as probes of the highredshift universe is that they are rare, and hence their detection requires surveys that cover a wide area of the sky (see the vertical axis in Figs. 2-4). The simplest strategy for identifying high-redshift afterglows is through all-sky surveys in the gamma-ray or X-ray regimes. In particular, detection of high-redshift sources will become feasible with the high trigger rate provided by the forthcoming Swift satellite, to be launched in 2003. Swift is expected to observe and localize $\sim 300$ GRBs per year and to repoint within 20-70 s its on-board X-ray and UV-optical instrumentation for continued afterglow studies. The high-resolution GRB coordinates obtained by Swift will be transmitted to Earth within $\sim 50 \mathrm{~s}$. Deep follow-up observations will then become feasible from the ground or using the highly sensitive infrared instruments on board the Next Generation 

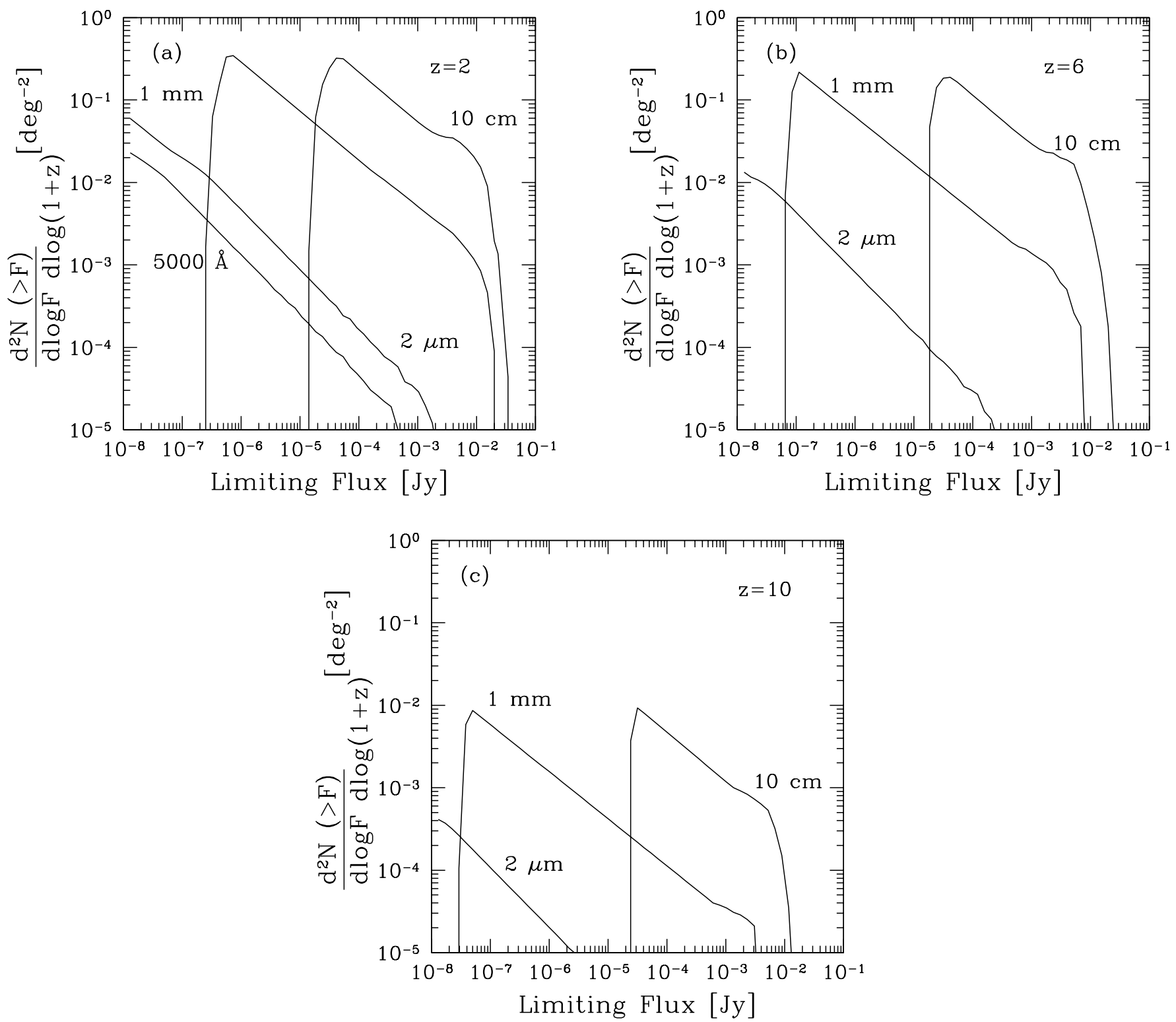

FIG. 4. - (a) Number count distribution per logarithmic flux and redshift interval. The observed wavelengths are, from the right to the left, $\lambda_{\text {obs }}=10 \mathrm{~cm}, 1$ $\mathrm{mm}, 2 \mu \mathrm{m}$, and $5000 \AA$. The redshift bin of width 0.1 is centered at $z=2$. (b) As in (a) with a redshift bin of width 0.1 centered at $z=6$. (c) As in $(a)$ with a redshift bin of width 0.1 centered at $z=10$.

Space Telescope, scheduled for launch in 2008. Swift is sufficiently sensitive to trigger on the gamma-ray emission from GRBs at redshifts $z \gtrsim 10$ (Lamb \& Reichart 2000). We note that even if some GRBs occur outside galaxies, the intergalactic medium would be sufficiently dense to produce an afterglow at these high redshifts since its mean density is $\gtrsim 10^{-4}[(1+z) / 10]^{3} \mathrm{~cm}^{-3}$.

The effect of beaming is expected to be minor for sources with millimeter and radio fluxes $\lesssim 10^{-5}$ Jy since the number counts at these fluxes are dominated by sources that are in the subrelativistic phase of their expansion and for which the energy output in the explosion is already isotropized (see Figs. $6 b$ and $6 c$ ).

For a characteristic energy output of GRBs of $\sim 10^{52}$ ergs, our model implies that at any time there should be $\sim 15$ GRBs from redshifts $z \gtrsim 5$ across the sky, which are brighter than $100 \mathrm{nJy}$ at an observed wavelength of $2 \mu \mathrm{m}$. The Next Generation Space Telescope will be able to measure the spectra of these sources. Prior to reionization, the spectrum of GRB afterglows might reveal the long sought after Gunn-Peterson trough (Gunn \& Peterson 1965), due to absorption by the neutral intergalactic medium.

We thank F. Haardt for providing us the IGM opacity curves and J. Bagla and R. Croft for useful discussions. B. C. thanks the CfA predoctoral fellowship program for support during the course of this work. This work was also supported in part by NASA grants NAG 5-7039 and NAG 5-7768, NSF grant 9900077 , and a grant from the Israel-US BSF. 

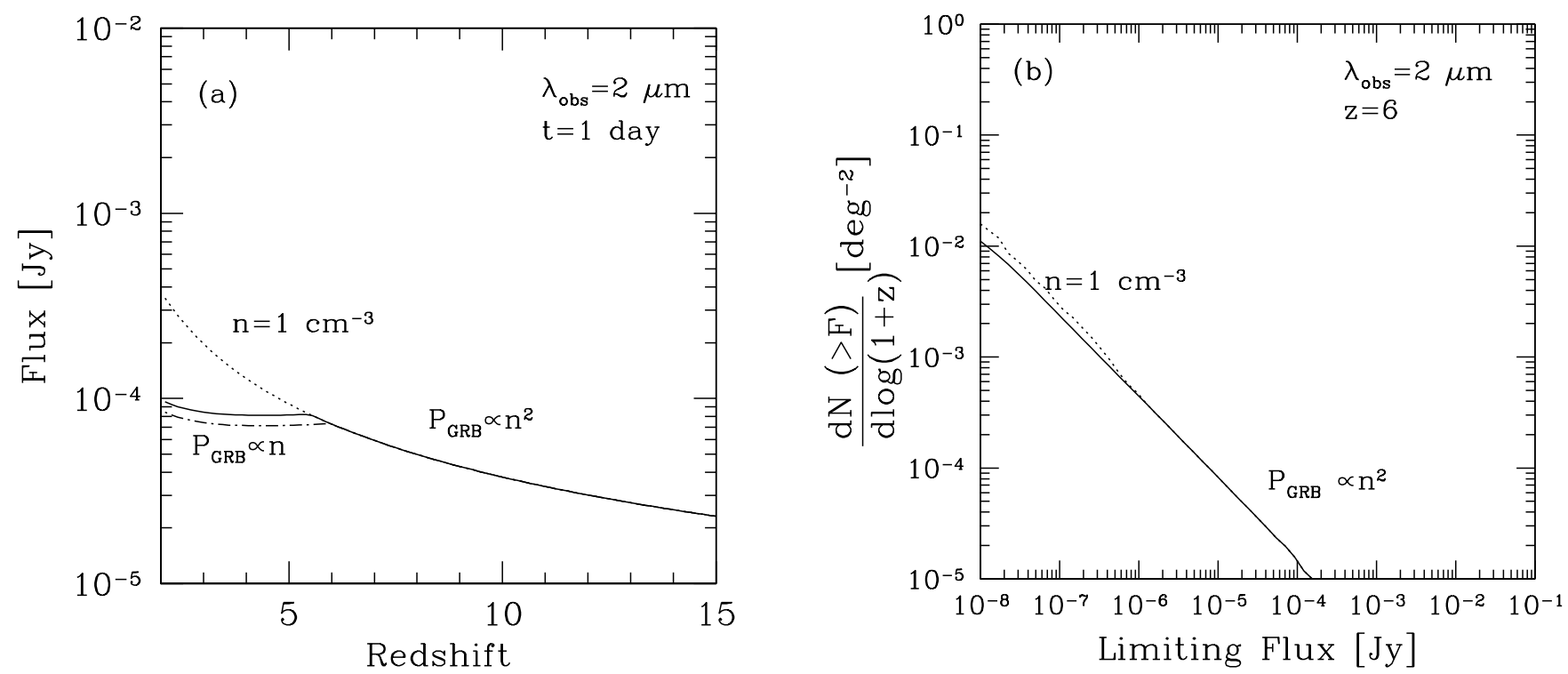

FIG. 5.-(a) Observed flux for a GRB hosted by a "typical" halo (having the mean mass) as a function of redshift (see text for details). The flux is shown for the reference observed wavelength $2 \mu \mathrm{m}$ and an observation time of 1 day after the GRB. The different curves correspond to different ambient densities, namely, a constant density of $1 \mathrm{~cm}^{-3}$ (dotted line) and density profiles of galactic disks with $P_{\mathrm{GRB}} \propto n^{2}$ (solid line; standard reference case) and with $P_{\mathrm{GRB}} \propto n$ (dot-dashed line). (b) Contribution to the total number count of GRBs from a redshift bin of width 0.1 centered at $z=6$ for an observed wavelength of $2 \mu$ m. Notation is the same as in $(a)$.

\section{APPENDIX}

\section{EVOLUTION OF THE GAS DENSITY IN GALACTIC DISKS}

The phenomenological modeling of many afterglow light curves implies values of the ambient medium density $\sim 1 \mathrm{~cm}^{-3}$, which are of order the mean density of the interstellar medium of disk galaxies (Waxman 1997; Wijers \& Galama 1999; Mészáros 1999). In popular hierarchical models of galaxy formation (Kauffmann, White, \& Guiderdoni 1993; Mo, Mao, \& White 1998), the mass and size of galactic disks evolves with redshift (Barkana \& Loeb 2000). Hence, in modeling the statistical properties of GRBs within disk galaxies at different redshifts, we need to follow the evolution of the average density of the interstellar gas within these galaxies as a function of cosmic time.

We model a disk galaxy as a gaseous disk embedded within a dark matter halo. The disk is assumed to be self-gravitating and radially exponential with a scale radius $r_{d}$. Its scale height at any radius is dictated by the balance between its self-gravity and the gas pressure. For simplicity, we assume the disk to be isothermal. Its mass density profile is then (Spitzer 1942)

$$
\rho(\zeta, r)=\rho_{0} e^{-r / r_{d}} \operatorname{sech}^{2}(\xi / \sqrt{2}),
$$

where $\zeta$ and $r$ are the vertical and radial coordinates of the disk, respectively; $\rho_{0}=$ const; $\xi=\zeta\left(4 \pi G \rho_{0} e^{-r / r_{d}} / c_{s}^{2}\right)^{1 / 2}$; and $c_{s}$ is the effective sound speed (including the possible contribution from turbulence). We adopt a sound speed of $c_{s} \approx 10 \mathrm{~km} \mathrm{~s}{ }^{-1}$, corresponding to a temperature of $\sim 10^{4} \mathrm{~K}$ below which atomic cooling is highly inefficient. At redshifts $z \lesssim 15$ the formation of galaxies with virial temperatures below $10^{4} \mathrm{~K}$ is expected to be suppressed because of various feedback effects (Barkana \& Loeb 1999; Ciardi et al. 2000).

The scale radius of the disk is dictated by the angular momentum per unit mass of the gas and can be expressed as (Mo et al. 1998)

$$
r_{d}=\frac{1}{\sqrt{2}} \frac{j_{d}}{m_{d}} \lambda r_{\mathrm{vir}}
$$

Here $j_{d}=J_{d} / J$ and $m_{d}=M_{d} / M$, where $J$ and $M$ are the halo angular momentum and mass and the subscript $d$ refers to the disk; $\lambda$ and $r_{\text {vir }}$ are the halo spin parameter and virial radius, respectively. We assume that the disk mass fraction is the cosmic baryonic fraction, $M_{d} / M=\left(\Omega_{b} / \Omega_{0}\right)$, since gas cooling is efficient at the high redshifts of interest. The characteristic size distribution of local galactic disks is obtained by adopting $j_{d} / m_{d}=1$ (Fall \& Efstathiou 1980). Based on numerical simulations, we parameterize the distribution of the spin parameter $\lambda$ by a lognormal form (Mo et al. 1998 and references therein)

$$
p(\lambda) d \lambda=\frac{1}{\sigma_{\lambda} \sqrt{2 \pi}} \exp \left[-\frac{\ln ^{2}(\lambda / \bar{\lambda})}{2 \sigma_{\lambda}^{2}}\right] \frac{d \lambda}{\lambda},
$$



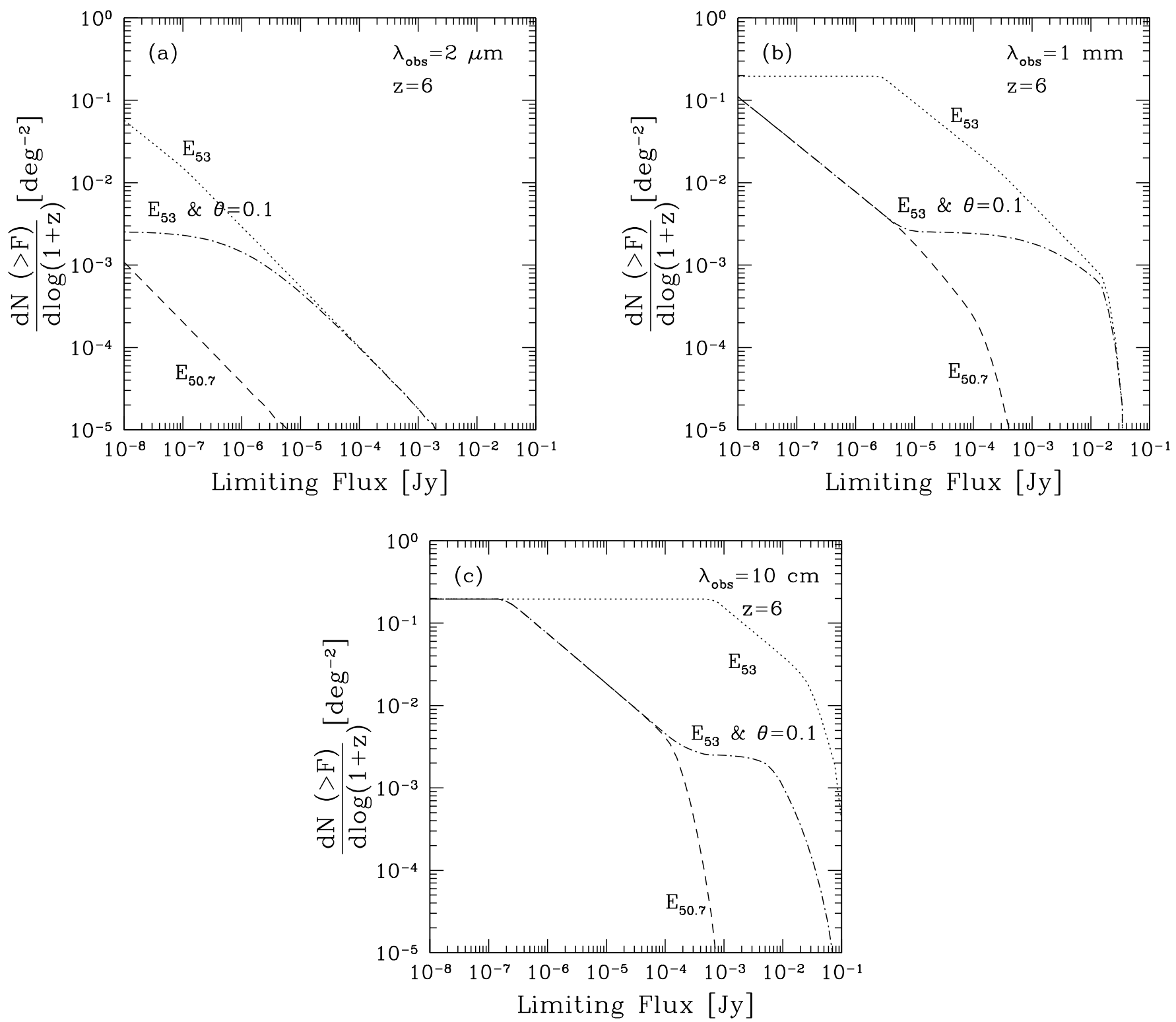

FIG. 6. - (a) Contribution to the total number count of GRBs from a redshift bin of width 0.1 centered at $z=6$ for an observed wavelength of $2 \mu \mathrm{m}$. The different curves refer to different energy outputs: $E=10^{53} \mathrm{ergs}$ (labeled $E_{53} ;$ dotted line) and $5 \times 10^{50} \mathrm{ergs}=10^{50.7}$ ergs (labeled $E_{50.7} ;$ dashed line). Two of the curves refer to a beamed explosion with an equivalent isotropic energy output of $10^{53} \mathrm{ergs}$ and a beaming angular radius of $\theta=0.1$ (dot-dashed line; see text for details). The actual energy output in this case is $5 \times 10^{50}$ ergs. (b) As in $(a)$ but for the reference observed wavelength of $1 \mathrm{~mm}$. (c) As in $(a)$ but for the reference observed wavelength of $10 \mathrm{~cm}$.

with $\bar{\lambda}=0.05$ and $\sigma_{\lambda}=0.5$. The halo virial radius is given by (Barkana \& Loeb 1999)

with $\delta_{c}=18 \pi^{2}$ and

$$
r_{\mathrm{vir}}=7.56\left[h^{-2} \frac{M}{10^{8} M_{\odot}} \frac{\Omega(z)}{\Omega_{m}} \frac{200}{\delta_{c}}\right]^{1 / 3}(1+z)^{-1} \mathrm{kpc}
$$

$$
\Omega(z)=\frac{\Omega_{m}(1+z)^{3}}{\Omega_{m}(1+z)^{3}+\Omega_{\Lambda}+\left(1-\Omega_{m}-\Omega_{\Lambda}\right)(1+z)^{2}} .
$$

The total mass of the disk, $M_{d}$, can be derived by integrating equation (A1). This yields the relation

$$
\rho_{0}=\frac{G M_{d}^{2}}{128 \pi c_{s}^{2} r_{d}^{4}} .
$$

The star formation rate depends on the local surface density of the gaseous disk. Schmidt's law implies that the star formation rate is proportional to the local surface density of the disk to the power of 1-2 (Kennicutt 1998). Hence, we first model the probability for a GRB occurrence within a volume element $2 \pi r d r d \zeta$ as being proportional to the density squared, $P_{\mathrm{GRB}}=\mathscr{A} n^{2}(\zeta, r)$, while in $\S 3.3$ we consider the sensitivity of our results to other power-law scalings. The proportionality constant $\mathscr{A}$ is chosen to normalize the integral of $P_{\mathrm{GRB}}$ to unity. Consequently, we can write the average of the ambient 
density probed by GRBs as

$$
\langle\rho\rangle=\int_{0}^{\infty} \int_{0}^{\infty} \rho(\zeta, r) P_{\mathrm{GRB}} 2 \pi r d r d \zeta=0.29 \rho_{0} .
$$

The number density of atoms in the medium is thus $\langle n\rangle=\langle\rho\rangle / \mu m_{p}$, where $\mu$ is the mean molecular weight. Similarly, we can derive $\langle\sqrt{n}\rangle$ and $\langle 1 / n\rangle$ and substitute them into equations (3)-(5). Even if the central engine of all GRBs were standard, the dependence of the ambient medium density on the properties (mass and size) of the host galaxy would have introduced significant scatter and evolution to the flux distribution of GRB afterglows.

\section{REFERENCES}

Bahcall, N. A., Ostriker, J. P., Perlmutter, S., \& Steinhardt, P. J. 1999, Science, 284, 1481

Barkana, R., \& Loeb, A. 1999, ApJ, 523, 54 2000, ApJ, 531, 613

Blain, A. W., \& Natarajan, P. 2000, MNRAS, 312, L35

Blandford, R. D., \& McKee, C. F. 1976, Phys. Fluids, 19, 1130

Bloom, J. S., et al. 1999, Nature, 401, 453

Böttcher, M., \& Dermer, C. D. 2000, ApJ, 529, 635

Chevalier, R. A., \& Li, Z. Y. 2000, ApJ, 536, 195

Chiu, W. A., \& Ostriker, J. P. 2000, ApJ, 534, 507

Ciardi, B., Ferrara, A., Governato, F., \& Jenkins, A. 2000, MNRAS, 314, 611

Costa, E., et al. 1997, Nature, 387, 783

Dai, Z. G., \& Lu, T. 1998, MNRAS, 298, 87

Eichler, D., Livio, M., Piran, T., \& Schramm, D. N. 1989, Nature, 340, 126

Fall, S. M., \& Efstathiou, G. 1980, MNRAS, 193, 189

Frail, D. A., et al. 1997, Nature, 389, 261

Frail, D. A., Waxman, E., \& Kulkarni, S. R. 2000, ApJ, 537, 191

Freedman, D. L., \& Waxman, E. 1999, preprint (astro-ph/9912214)

Fruchter, A. S., et al. 1999, ApJ, 516, 683

Gnedin, N. Y. 2000, ApJ, 535, 530

Gunn, J. E., \& Peterson, B. A. 1965, ApJ, 142, 1633

Haardt, F., \& Madau, P. 1996, ApJ, 461, 20

Haiman, Z., \& Loeb, A. 1997, ApJ, 483, 21

1998, ApJ, 503, 505 1999, ApJ, 521, L9

Haiman, Z., Rees, M. J., \& Loeb, A. 1997, ApJ, 476, 458

Huang, Y. F., Dai, Z. G., \& Lu, T. 1998, A\&A, 336, L69

Kauffmann, G., White, S. D. M., \& Guiderdoni, B. 1993, MNRAS, 264, 201

Kennicutt, R. C., Jr. 1998, ApJ, 498, 541

Krumholz, M., Thorsett, S. E., \& Harrison, F. A. 1998, ApJ, 506, L81

Kulkarni, S. R., et al. 1999, Nature, 398, 389 2000, preprint (astro-ph/0002168)

Kumar, P. 1999, ApJ, 523, L113

Kumar, P., \& Piran, T. 2000, ApJ, 535, 152

Lamb, D. Q., \& Reichart, D. E. 2000, ApJ, 536, 1

Lilly, S. J., Le Fèvre, O., Hammer, F., \& Crampton, D. 1996, ApJ, 460, L1

Loeb, A. 1999, in ASP Conf. Ser. 193, The Hy-Redshift Universe: Galaxy Formation and Evolution at High Redshift, ed. A. J. Bunker \& W. J. M. van Breugel (San Francisco: ASP), 586

MacFadyen, A. I., \& Woosley, S. E. 1999, ApJ, 524, 262
Madau, P., et al. 1996, MNRAS, 283, 1388

Madau, P., Haardt, F., \& Rees, M. J. 1999, ApJ, 514, 648

Madau, P., \& Pozzetti, L. 2000, MNRAS, 312, L9

Medvedev, M. V., \& Loeb, A. 1999, ApJ, 526, 697

Meegan, C. A., et al. 1992, Nature, 355, 143

Mészáros, P. 1999, preprint (astro-ph/9912474)

Mészáros, P., \& Rees, M. J. 1999, MNRAS, 306, L39

Mészáros, P., Rees, M. J., \& Wijers, R. A. M. J. 1998, ApJ, 499, 301

Metzger, M. R., et al. 1997, Nature, 387, 878

Miralda-Escudé, J., Haehnelt, M., \& Rees, M. R. 2000, ApJ, 530, 1

Miralda-Escudé, J., \& Rees, M. R. 1998, ApJ, 497, 21

Mo, H. J., Mao, S., \& White, S. D. M. 1998, MNRAS, 295, 319

Mochkovitch, R., Hernanz, M., Isern, J., \& Martin, X. 1993, Nature, 361, 236

Paczyński, B. 1991, Acta Astron., 41, 257

. 1998, ApJ, 494, L45

Padmanabhan, T. 1993, Structure Formation in the Universe (Cambridge: Cambridge Univ. Press)

Panaitescu, A., \& Mészáros, P. 1999, ApJ, 526, 707

Press, W. H., \& Schechter, P. 1974, ApJ, 187, 425

Reichart, D. E. 1999, ApJ, 521, L111

Rhoads, J. E. 1999a, ApJ, 525, 737 1999b, A\&AS, 138, 539

Sahu, K., et al. 1997, ApJ, 489, L127

Sari, R., Piran, T., \& Narayan, R. 1998, ApJ, 497, L17

Sedov, L. I. 1959, Similarity and Dimensional Methods in Mechanics (New York: Academic)

Spitzer, L., Jr. 1942, ApJ, 95, 329

Stern, D., \& Spinrad, H. 1999, PASP, 111, 1475

Taylor, G. I. 1950, Proc. R. Soc. London A, 201, 159

Totani, T. 1997, ApJ, 486, L71

Usov, V. V. 1992, Nature, 357, 472

Valageas, P., \& Silk, J. 1999, A\&A, 347, 1

van Paradijs, J., et al. 1997, Nature, 386, 686

Waxman, E. 1997, ApJ, 489, L33

Wei, D. M., \& Lu, T. 1999, preprint (astro-ph/9912063)

Wijers, R. M. J., Bloom, J. S., Bagla, J. S., \& Natarajan, P. 1998, MNRAS, 294, L13

Wijers, R. A. M. J., \& Galama, T. J. 1999, ApJ, 523, 177

Wood, K., \& Loeb, A. 1999, ApJ, accepted (preprint astro-ph/9911316)

Woods, E., \& Loeb, A. 1999, preprint (astro-ph/9907110) 